УДК 338.32

$10.17213 / 2075-2067-2020-3-73-78$

\title{
АЛГОРИТМ ФОРМИРОВАНИЯ РАСПИСАНИЯ РАБОТЫ ЦЕХА МЕХАНИЧЕСКОЙ ОБРАБОТКИ МАШИНОСТРОИТЕЛЬНОГО ПРЕДПРИЯТИЯ
}

\author{
(C) 2020 г. П. В. Овчинников, Д. А. Гиммельберг, П. П. Савельчев
}

\author{
Южно-Российский государственный политехнический университет (НПИ) \\ имени М. И. Платова, г. Новочеркасск, Россия
}

Целью исследования является разработка эвристического алгоритма для формирования расписания работы станочного оборудования, расположенного в цехе механической обработки крупного машинострочтельного предприятия.

Методологическую базу исследования составляют работы ведущих отечественных ученых в сфере планирования производства, автоматизации производства, разработки и применения оптимизационных алгоритмов. Особое значение имеет опьт, накопленный исследователями, занимающимися практическим внедрением инструментов моделирования производственных прочессов.

Результаты исследования. Разработан решающий алгоритм, позволяющий составить расписание работы цеха. При этом обеспечивается минимизация отклонения сроков фактического производства изделий от плановых значений.

Перспектива исследования заключается в формализации параметров переналадки станочного оборудования при замене обрабатываемой сборочной единиць.

Ключевые слова: расписание работы; организация производства; эвристический алгоритм; минимизация отклонений.

\section{ALGORITHM FOR THE FORMATION OF THE WORK SCHEDULE OF THE MACHINING SHOP OF A MACHINE-BUILDING ENTERPRISE}

\author{
(C) 2020 P. V. Ovchinnikov, D. A. Gimmelberg, P. P. Savelchev
}

Platov South Russian State Polytechnic University (NPI), Novocherkassk, Russia

The target of the study is to develop a heuristic algorithm for forming a schedule of work of machine equipment located in the machining shop of a large machine-building enterprise.

The research methodology is based on the work of leading Russian scientists in the field of production planning, automation of production, development and application of optimization algorithms. Of particular importance is the experience gained by researchers involved in the practical implementation of tools for modeling production processes.

The results of the study. A decisive algorithm has been developed to schedule the workshop. This minimizes the deviation of the timing of the actual production of products from planned values.

The prospect of the study is to formalize the readjustment parameters of the machine equipment when replacing the machined assembly unit.

Key words: work schedule; organization of production; heuristic algorithm; minimization of deviations. 
Введение. В настоящей работе предпринята попытка разработки эвристического алгоритма для формирования расписания работы станочного оборудования, расположенного в цехе механической обработки крупного машиностроительного предприятия. Алгоритм требуется составить таким образом, чтобы обеспечить минимальное отклонение фактического выпуска сборочных единиц от плана выпуска данного цеха. Методологическую базу составляют исследования ведущих отечественных ученых в сфере планирования производства [5, 8], автоматизации производства $[2,3]$, разработки и применения оптимизационных алгоритмов $[1,4]$. Особое значение имеет опыт, накопленный исследователями, занимающимися практическим внедрением инструментов моделирования производственных процессов $[6,7,9]$.

Постановка задачи. Пусть цех механической обработки предприятия дискретного машиностроения осуществляет обработку заданной номенклатуры деталей. Каждая деталь, пройдя заданную последовательность этапов обработки в рамках цеха, преобразуется в сборочную единицу, которая затем поступает в сборочный цех. Потребность сборочного цеха в сборочных единицах определяет план цеха механической обработки. План представляет собой набор маршрутных карт (далее - МК), включающих в себя: наименование требуемой сборочной единицы, количество по плану, момент времени, в который требуется поступление соответствующей сборочной единицы в сборочный цех. Деталь в процессе обработки проходит последовательность рабочих центров, объединенных в группы по функциональному признаку. Процесс взаимодействия цеха механической обработки и сборочного цеха представлен на рисунке 1.

Введем следующие обозначения:

$-\left\{M K_{i}\right\}, i=\overline{1, M}$ - множество идентификаторов (id) маршрутных карт, $M$ - общее число маршрутных карт;

$-\left\{R C_{j}\right\}, j=\overline{1, N}$ - множество идентификаторов (id) рабочих центров (РЦ), $N$ общее число рабочих центров;

- $\left\{G R C_{l}\right\}, l=\overline{1, L}$ - множество идентификаторов (id) групп рабочих центров (ГРЦ), $L$ - общее число групп рабочих центров. Для каждого $j$-го РЦ и $l$-го ГРЦ можно установить соответствие (входит ли РЦ в ГРЦ);

- $\left\{T_{f}\right\}, f=\overline{1, F}$ - множество моментов времени, для которых определен план окончания выполнения маршрутных карт.

Для каждой маршрутной карты $M K_{i}$ вводим:

— переменную $K M K_{i}$ - количество деталей, входящих в $i$-ю маршрутную карту;

- векторную переменную $\overline{C M K_{i}}=$ $=\left\{C M K_{i}^{1} \ldots C M K_{i}^{k_{i}}\right\}$, где $C M K_{i}^{1} \ldots C M K_{i}^{k_{i}}$ номера (идентификаторы, id) групп рабочих центров, задающие последовательность прохождения маршрутной карты $M K$;

- $k_{i}$ - количество рабочих центров, которые проходит $i$-я маршрутная карта;

- векторную переменную $\overline{T M K_{i}}=$ $=\left\{T M K_{i}^{1} \ldots T M K_{i}^{k_{i}}\right\}, \quad$ где $T M K_{i}^{1} \ldots T M K_{i}^{k_{i}^{i}}-$ штучное время обработки детали на рабочих центрах, входящих в ГРЦ $C M K_{i}^{1} \ldots C M K_{i}^{k_{i}}$.

Векторы $\overline{C M K_{i}}$ и $\overline{T M K_{i}}$ имеют вариативную размерность, при этом для одной маршрутной карты $M K_{i}$ их размерность совпадает и составляет $k_{i}$.

Для каждой маршрутной карты рассчитаем продолжительность ее обработки на со-

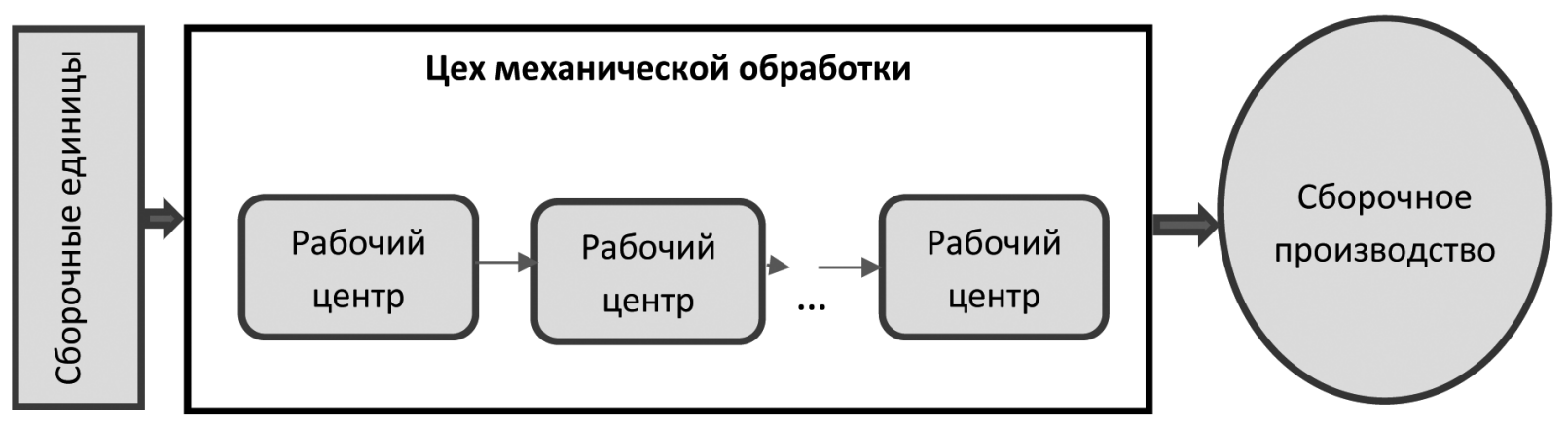

Рис. 1. Взаимодействие цеха механической обработки и сборочного цеха 
ответствующих рабочих центрах, получив вектор

$$
\begin{aligned}
& \overline{V M K_{i}}=\left\{V M K_{i}^{1} \ldots V M K_{i}^{k_{i}}\right\}, \\
& \text { где } V M K_{i}^{k}=K M K_{i} \cdot T M K_{i}^{k} .
\end{aligned}
$$

Рассчитаем общую продолжительность исполнения каждой маршрутной карты:

$$
V M K_{i}=\sum_{k=1}^{k_{i}} V M K_{i}^{k} .
$$

Для каждого рабочего центра вводим переменную «состояние рабочего центра»:

$S R C_{j}=0$, если $j$-й РЦ простаивает,

$S R C_{j}=M K_{i}, j$-й РЦ загружен $i$-й маршрутной картой,

$S R C_{j}=1$, если $j$-й РЦ недоступен.

Для каждой маршрутной карты вводим переменную «состояние маршрутной карты» $\overline{S M K_{i}}=\left\{S M K_{i}^{1} \ldots S M K_{i}^{k_{i}}\right\}, S M K_{i}^{k}=0$, если обработка на соответствующей ГРЦ не завершена, $S M K_{i}^{k}=S M K_{i}^{k}$, если обработка на соответствующей ГРЦ завершена.

Для каждого РЦ задана интервальная переменная $\mathcal{T}_{j}=\left\{\mathcal{T}_{j}^{1}, \ldots \mathcal{T}_{j}^{q_{j}}\right\}$, где $\mathcal{T}_{j}^{q}-$ временные интервалы доступности $j$-го РЦ.

Для каждого РЦ задаем параметр $T R C_{j}-$ общее время занятости рабочего центра. Представим план производства в виде набора векторов $\left\{\overline{P_{f}}\right\}, f=\overline{1, F}$, где $\overline{P_{f}}-$ план производства, соответствующий моменту $T_{f}$

Вектор $\overline{P_{f}}=\left\{p_{f}^{i}\right\}, i=\overline{1, M}$.

$p_{f}^{i}=0$, если в данный момент выпуск $i$-й MK не запланирован,

$p_{f}^{i}=M K_{i}$, если в данный момент выпуск $i$-й МК запланирован.

Вводим счетчик времени $\tau$. Принимаем шаг $\tau-1$ секунда.

Последовательность действий по реализации алгоритма. Для $f=1$ проводим анализ вектора $\overline{P_{f}}$. Ранжируем длительности выполнения всех маршрутных карт, запланированных к данному моменту времени, т.е. упорядочиваем по возрастанию величины $V M K_{i}$ для всех $i$, для которых выполняется $p_{f}^{i} \neq 0$. Распределение маршрутных карт по РЦ начинаем с маршрутной карты, для которой выполняется условие $\max V M K_{i}$. Фиксируем $M K_{i}-\mathrm{id}$ данной маршрутной карты.

В начальный момент времени $(\tau=0)$ принимаем:

- все рабочие центры не загружены, т.е. $S R C_{j}=0$ для всех $j$
- все маршрутные карты находятся в начальной стадии (ни один РЦ ни одной маршрутной картой не пройден), т.е. $S M K_{i}^{k}=0$ для всех $i$ и всех $k$.

Задаем цикл перебора по всем $j=\overline{1, N}$. Для каждого $R C_{j}$ определяем соответствующий ему $G R C_{l}$.

Если $G R C_{l}=C M K_{i}^{1}$ (т.е. id свободного ГРЦ совпал с id ГРЦ, стоящего первым в маршрутной карте максимальной длительности), то производим назначение маршрутной карты $M K_{i}$ на $j$-й РЦ. Для данного РЦ $S R C_{j}$ принимает значение $M K_{i}$. Фиксируется значение переменной $\tau_{i}^{j}$ - момент назначения карты $i$ на РЦ $j$. В противном случае переходим к следующему РЦ.

Аналогичную операцию проводим для всех маршрутных карт в порядке убывания параметра $V M K_{i}$. Если $S R C_{j} \neq 0$, то возможность назначения на данный РЦ не рассматривается.

В случае, если после перебора всех $M K_{i}$ (при таких $i$, что $p_{f}^{i} \neq 0$ ) остались незанятые РЦ (т.е. $R C_{j}$ такие, что $S R C_{j}=0$ ) переходим к распределению плана производства для $f=2$ (и далее).

Запускаем счетчик времени $\tau$ с шагом в 1 секунду. Можно сказать, что $\tau_{t}=\tau_{t-1}+1$.

На каждом шаге для всех $R C_{j}$ проверяем следующие условия (через цикл по индексу $j$ ):

- $\tau \in \mathcal{T}_{j}^{q}$ - временным интервалам доступности $R C_{j}$. Если это условие не выполняется, то принимается $S R C_{j}=1$;

- если для данного $j$ выполняется $R C$ равно некоторому $M K \ldots$ :.., то проверяется условие $\tau \leq T M K_{i}^{1}$. Если $\tau>T M K_{i}^{1}$, то рабочий центр с id $R C_{j}$ высвобождается, т.е. $S R C_{j}:=0$.

Высвобождение РЦ означает, что на нем закончена обработка маршрутной карты с id $M K^{\phi}{ }_{i}$. Для этой маршрутной карты принимаем $S M K_{i}^{1}=C M K_{i}^{1}$.

Для высвободившегося рабочего центра проводим операцию назначения маршрутной карты. Маршрутная карта, обработка которой закончена на центре $R C_{j}$, получает возможность назначения на следующий РЦ. Для этого по всем центрам, для которых выполняется $S R C_{j}=0$, проверяется условие $C M K_{i}^{2}=G R C_{l}$, где $G R C_{l}$ - id ГРЦ, которому соответствует $R C_{j}$. При выполнении данного условия фиксируется $\tau_{i}^{j}$ - момент назначения карты $i$ на РЦ с номером $j$. 
Задача распределения маршрутных карт по рабочим центрам решается каждый ра3, когда высвобождается тот или иной РЦ. Непрерывно отслеживается для каждого РЦ выполнение условия $\tau \in \mathcal{T}_{j}^{q}$ (РЦ находится во временном интервале доступности).

В момент времени $T_{1}$ (первая временная точка плана производства) проверяется его выполнение. Для всех $M K_{i}$, таких, что $p_{f}^{i} \neq 0$, проводится анализ вектора $\overline{S M K_{i}}=\left\{S M K_{i} \ldots S M K_{i}^{k_{i}}\right\}$.

Если все компоненты вектора ненулевые, то $i$-я маршрутная карта выполнена.

В случае невыполнения маршрутной карты определяются номера ГРЦ $\left(C M K_{i}^{k}\right)$, которые данная карта не прошла. Оценивается возможность добавления фонда рабочего времени в доступный фонд рабочего времени РЦ, для которого выполняется $R C_{j} \in G R C_{l}\left(G R C_{l}=C M K_{i}^{k}\right)$.

Добавление фонда рабочего времени производится путем расширения интервалов $\mathcal{T}_{j}^{q}$ (сдвигается верхняя граница интервала на величину, кратную $T M K_{i}^{k}$ ).

Производится переформирование плана распределения МК.

В результате реализации предложенного алгоритма будет получен набор параметров $\left\{M K_{i}, R C_{j}, \tau_{i}^{j}\right\}$ - идентификаторы маршрутной карты, рабочего центра и момент назначения карты для обработки.

\section{Литература}

1. Гладков Л.А., Курейчик В. В., Курейчик B.M. и др. Биоинспирированные методы в оптимизации: монография. - М.: Физматлит, 2009. - 384 с.

2. Ииккович Э.Л. Современные проблемы и методы эффективной автоматизации производства на российских предприятиях технологического и энергетического типа.// Автоматизация и управление в ЦБП, ЛПК и энергетике. / Материалы XI Международной научно-технической конференции. Петрозаводск. - 2016. - С. 14-18.

3. Лопухов И. Коммуникационные технологии умного предприятия в рамках концепции Индустрия 4.0 и Интернета вещей // Современные технологии автоматизации. 2015. 一 №2. - С. 36-45.

4. Курейчик В. М., Каплунов Т.Г. Адаптивный генетический алгоритм на основе нечетких правил. // Известия ЮФУ. Тех- нические науки. — 2018. — №5 (199). C. 26-34.

5. Мауэргауз Ю. Е. «Продвинутое» планирование и расписания (AP\&S) в производстве и цепочках поставок. - М.: Экономика, 2012. - 574 с.

6. Мохов В.А., Бородулина Е.Н. К вопросу о параметрической оптимизации роевых алгоритмов // Изв. ЮФУ. Техн. науки. 2014. - №4 (153). - С. 230-234.

7. Сычев В.А., Сычева Г.И. Современные подходы к организации и управлению промышленным производством. / Вестник Южно-Российского государственного технического университета (Новочеркасского политехнического института). Серия: Социально-экономические науки. - 2019. - №2. - С. 4-14.

8. Титов B. B. Оптимизация управления промышленной корпорацией. // Вопросы методологии и моделирования / Российская академия наук, Сибирское отделение, Институт экономики и организации промышленного производства. - Новосибирск, 2007.

9. Ткачев А. Н., Багдасарова М.В. Имитационное моделирование финансово-хозяйственной и производственной деятельности предприятий методами системной динамики.// Современные проблемы науки и образования. - 2014. — №5. - С. 371-375.

\section{References}

1. GladkovL.A., KurejchikV. V., Kurejchik V.M. at al. Bioinspirirovannye metody $\mathrm{v}$ optimizacii: monografija [Bioinspired methods in optimization: monograph]. - Moscow: Fizmatlit, 2009. - $384 \mathrm{p}$.

2. Ickovich Je. L. Sovremennye problemy i metody jeffektivnoj avtomatizacii proizvodstva na rossijskih predprijatijah tehnologicheskogo i jenergeticheskogo tipa [Modern problems and methods of effective automation of production at Russian enterprises of technological and energy type]. // Avtomatizacija i upravlenie v CBP, LPK i jenergetike [Automation and management in the Central Bank, LPC and power industry]. / Materialy XI Mezhdunarodnoj nauchno-tehnicheskoj konferencii [Proceedings of the XI International scientific and technical conference]. - Petrozavodsk. - 2016. - Pp. 14-18.

3. Lopuhov I. Kommunikacionnye tehnologii umnogo predprijatija $\mathrm{v}$ ramkah kon- 
cepcii Industrija 4.0 i Interneta veshhej [Smart enterprise communication technologies within the framework of industry 4.0 and the Internet of things] // Sovremennye tehnologii avtomatizacii [Modern automation technologies]. — 2015. №2. - Pp. 36-45.

4. Kurejchik V.M., Kaplunov T.G. Adaptivnyj geneticheskij algoritm na osnove nechetkih pravil [Adaptive genetic algorithm based on fuzzy rules]. // Izvestija JuFU. Tehnicheskie nauki [Izvestiya SFU. Technical science]. 2018. — №5 (199). — Pp. 26-34.

5. Maujergauz Ju. E. «Prodvinutoe» planirovanie i raspisanija (AP\&S) v proizvodstve i cepochkah postavok [«Advanced» planning and scheduling (AP\&S) in production and supply chains]. - Moscow: Jekonomika, 2012. - $574 \mathrm{p}$.

6. Mohov V.A., Borodulina E. N. K voprosu o parametricheskoj optimizacii roevyh algoritmov [On the question of parametric optimization of swarm algorithms] // Izv. JuFU. Tehn. Nauki [Technical Sciences]. — 2014. — №4 (153). Pp. 230-234.

7. Sychev V.A., Sycheva G.I. Sovremennye podhody $\mathrm{k}$ organizacii i upravleniju promyshlennym proizvodstvom [Modern approaches to the organization and management of industrial production]. / Vestnik Juzhno-Rossijskogo gosudarstvennogo tehnicheskogo universiteta (Novocherkasskogo politehnicheskogo instituta). Serija: Social'no-jekonomicheskie nauki [Bulletin of the South Russian state technical University (Novocherkassk Polytechnic Institute). Series: Socio-economic Sciences]. — 2019. №2. - Pp. 4-14.

8. Titov V.V. Optimizacija upravlenija promyshlennoj korporaciej [Optimization of industrial Corporation management]. // Voprosy metodologii i modelirovanija [Questions of methodology and modeling] / Rossijskaja akademija nauk, Sibirskoe otdelenie, Institut jekonomiki i organizacii promyshlennogo proizvodstva [Russian Academy of Sciences, Siberian branch, Institute of Economics and industrial production organization]. — Novosibirsk, 2007.

9. Tkachev A. N., Bagdasarova M.V. Imitacionnoe modelirovanie finansovo-hozjajstvennoj i proizvodstvennoj dejatel'nosti predprijatij metodami sistemnoj dinamiki [Simulation of financial, economic and production activities of enterprises using system dynamics methods]. // Sovremennye problemy nauki i obrazovanija [Modern problems of science and education]. 2014. — №5. — Pp. 371-375.

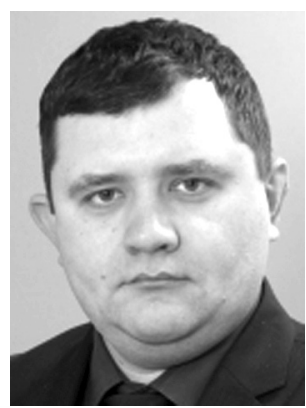

Овчинников Петр Вячеславович - кандидат экономических наук, исполняющий обязанности декана факультета инноватики и организации производства Южно-Российского государственного политехнического университета (НПИ) имени М.И. Платова.

Ovchinnikov Pyotr Vyacheslavovich - Candidate of Economic Sciences, Acting Dean, Faculty of Innovation and Production Organization, Platov South Russian State Polytechnic University (NPI).

346428, г. Новочеркасск, ул. Просвещения, 132 


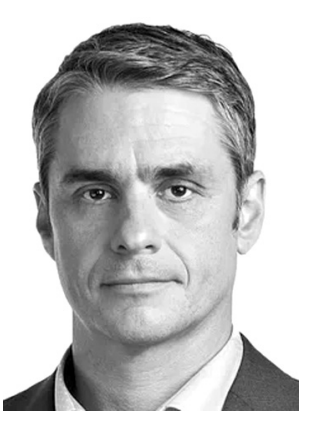

Гиммельберг Дмитрий Альбертович - аспирант кафедры «Производственный и инновационный менеджмент» ЮжноРоссийского государственного политехнического университета (НПИ) имени М. И. Платова.

Gimmelberg Dmitry Albertovich - Post-graduate Student, Department of Industrial and Innovation Management, Platov South Russian State Polytechnic University (NPI).

346428, г. Новочеркасск, ул. Просвещения, 132

132 Prosveshcheniya st., 346428, Novocherkassk, Russia

E-mail: d.gimmelberg@2050-integrator.com

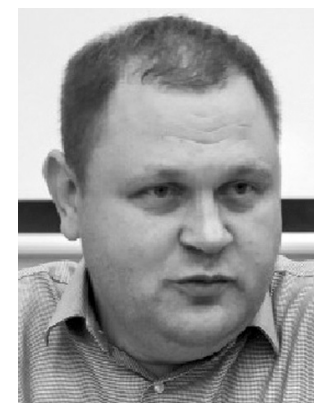

Савельчев Павел Петрович - аспирант кафедры «Производственный и инновационный менеджмент» Южно-Российского государственного политехнического университета (НПИ) имени М.И. Платова.

Savelchev Pavel Pyotrovich - Post-graduate Student, Department of Industrial and Innovation Management, Platov South Russian State Polytechnic University (NPI).

346428 , г. Новочеркасск, ул. Просвещения, 132

132 Prosveshcheniya st., 346428, Novocherkassk, Russia

E-mail: kz@kolomnadiesel.ru 